Portland State University

PDXScholar

\title{
A Pathway Linking Smart Growth Neighborhoods to Home-Based Pedestrian Travel
}

Steven R. Gehrke

Portland State University

Kelly J. Clifton

Portland State University, kclifton@pdx.edu

Follow this and additional works at: https://pdxscholar.library.pdx.edu/trec_seminar

Part of the Transportation Commons, Urban Studies Commons, and the Urban Studies and Planning Commons

Let us know how access to this document benefits you.

\section{Recommended Citation}

Gehrke, Steven R. and Clifton, Kelly J., "A Pathway Linking Smart Growth Neighborhoods to Home-Based Pedestrian Travel" (2017). TREC Friday Seminar Series. 105.

https://pdxscholar.library.pdx.edu/trec_seminar/105

This Book is brought to you for free and open access. It has been accepted for inclusion in TREC Friday Seminar Series by an authorized administrator of PDXScholar. Please contact us if we can make this document more accessible: pdxscholar@pdx.edu. 


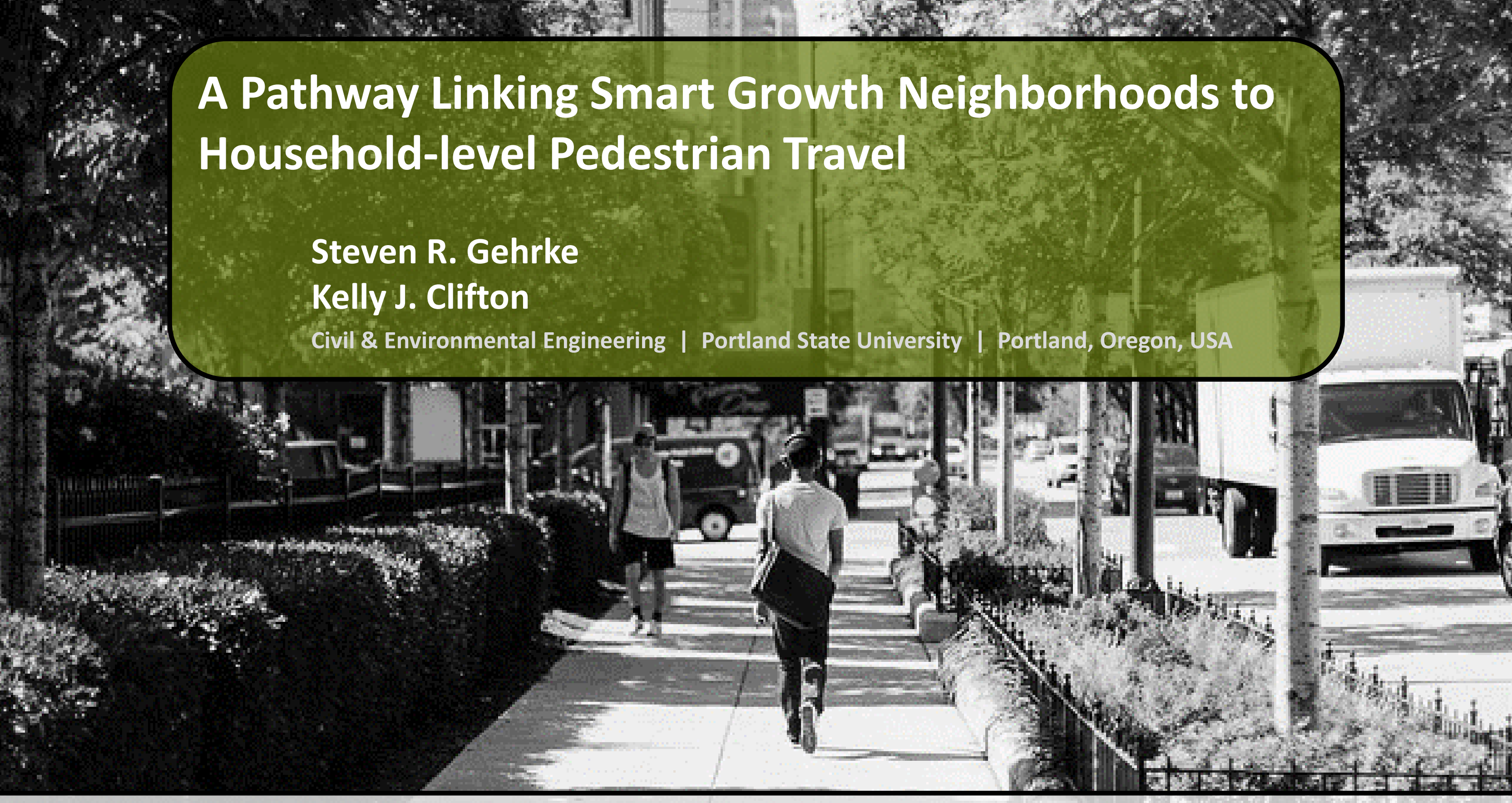


Land development patterns and urban design linked to travel behavior

- Smart growth policies and practice create activity-friendly, walkable communities

- Policy goal in 2009 Interagency Partnership for Sustainable Communities

Need to identify built environment indicators and set common standards

- Past active travel studies have adopted imperfect built environment measures

- Host of individual, societal, and contextual factors are hypothesized to predict walking behaviors for transportation and recreational purposes

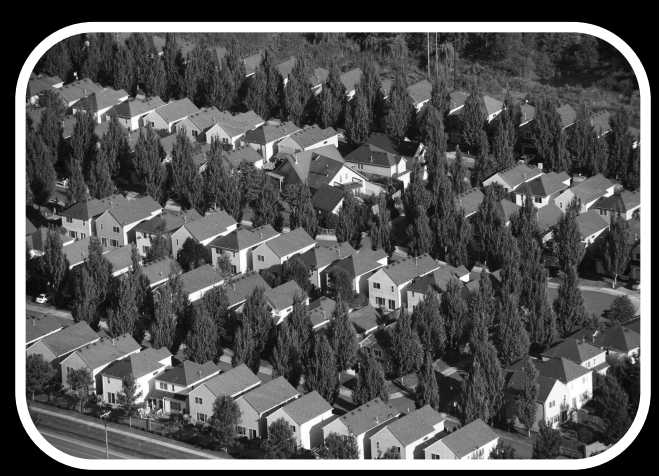

Compact Development

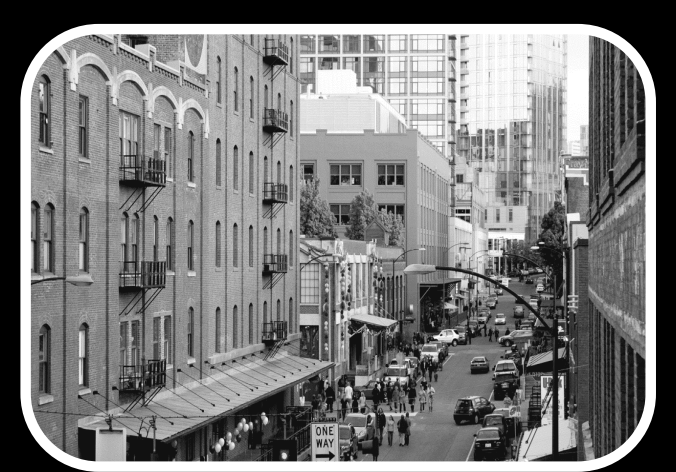

High Activity Density

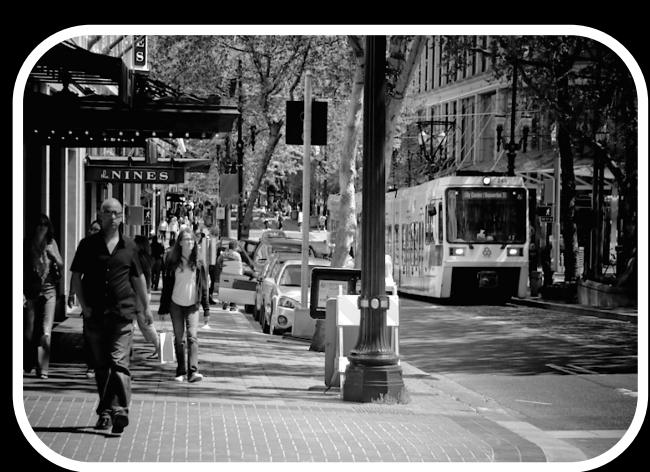

Walkable Design 
Introduce a multidimensional construct of the built environment

- Reflect several heralded tenants of smart growth development

- Offer insight into measurement selection and packaging of different elements

Propose framework and method linking this construct to pedestrian travel

- Simultaneously account for various and confounding determinants of walking

- Extend understanding of link between smart growth development and walking

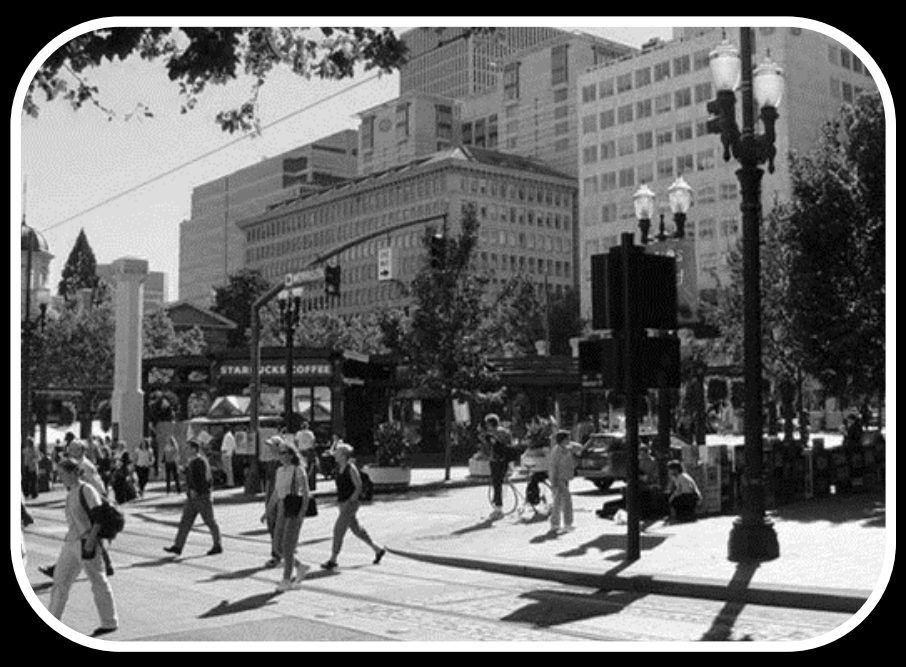


Individual perceptions of built environment

- Explore themes of neighborhood accessibility, arrangement and aesthetic, and sense of place to recognize their influence on auto ownership and mode choice

- Subject to reporting bias that may inflate connection and difficulty in translation

Objective measures of built environment

- Early studies explored limited number of indicators to reflect land use construct

- Recent studies test more indicators to examine short- and long-term decisions

Important gaps

- Few SEM studies exclusively reflect built environment with objective measures

- Studies largely examine built environment impact on auto-related outcomes 


\section{Conceptual framework}

\section{Built Environment}

Land Development Patterns

- Land Use Mix

- Density

Urban Design

- Arrangement

- Aesthetic

Transportation System

- Infrastructure

- Performance

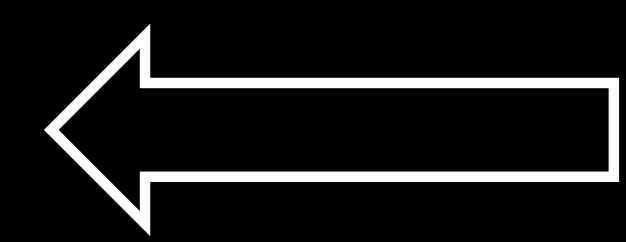

Sociodemographic, Economic, and Psychosocial

Individual

Household

Neighborhood

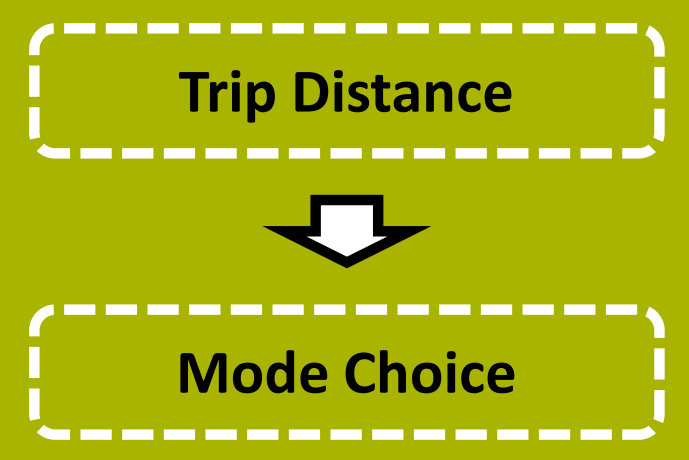

\section{Travel Behaviors and Patterns}

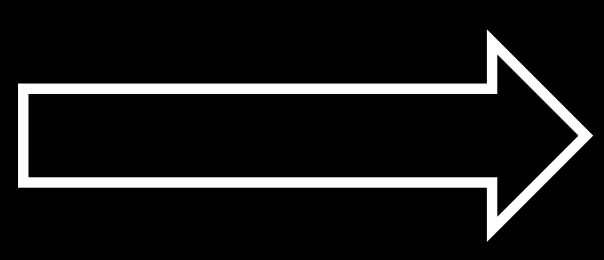

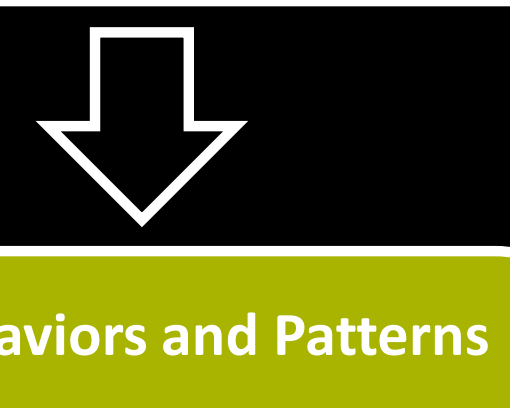

Trip Distance 


\section{Study area and sample}

- Multnomah, Clackamas, and Washington Counties in Oregon

- 2011 Oregon Household Activity and Travel Survey (OHAS)

- One-day travel diary for a study sample of 4,416 households

- Travel behavior and sociodemographic and economic information

\section{Built environment measurement}

- Set of 62 indicators measured within a one-mile areal buffer at home location

- Secondary data sources

- 2011 Portland Metro Regional Land Information System (RLIS)

- 2014 Longitudinal Employer-Household Dynamic (LEHD)

- 2010 US Census and Topologically Integrated Geographic Encoding and Referencing (TIGER) 


\section{Built environment measurement}

\section{Built Environment}

Land Development Patterns

- Land Use Mix

- Density

Urban Design

- Arrangement

- Aesthetic

Transportation System

- Infrastructure

- Performance

\section{Land Use Mix: Composition}

- Land use percent for nine land use types

- Land use entropy index

- Land use balance

- Activity-related complementarity

- Employment entropy

- Employment-population balance

- Retail employment-population balance

- Land use patches for nine land use types

\section{Land Use Mix: Configuration}

- Maximum patch size for nine land use types

- Maximum patch size (overall)

- Contagion index 


\section{Built environment measurement}

\section{Built Environment}

Land Development Patterns

- Land Use Mix

- Density

Urban Design

- Arrangement

- Aesthetic

Transportation System

- Infrastructure

- Performance

\section{Density}

- Population

- Housing units

- Employment

- Office jobs

- Retail jobs

- Industrial jobs

- Service jobs

- Entertainment jobs

- Total activity (population and employment) 


\section{Built environment measurement}

\section{Built Environment}

Land Development Patterns

- Land Use Mix

- Density

Urban Design

- Arrangement

- Aesthetic

Transportation System

- Infrastructure

- Performance

\section{Urban Design and Transportation System}

- Census blocks

- Street blocks

- Connected node ratio

- Alpha index

- Beta index

- Gamma index

- Three- and four-way intersections

- Cul-de-sacs

- Miles of primary, secondary, and local roads

- Percent of primary, secondary, and local roads

- Sidewalk coverage 
Analytic approach

\section{Zero-order correlation matrix}

- Eliminate associated measures that point toward concept redundancy

\section{Exploratory factor analysis}

- Identify sets of interrelated measures reflecting built environment dimensions

- Generate theoretic understanding of internal structure of measures

\section{Structural equation modeling}

- Confirmatory factor analysis

- Identify latent constructs of built environment reflecting multiple indicators

- Path analysis

- Simultaneously test for direct and indirect effects of built environment on pedestrian travel 


\section{Confirmatory factor analysis}

\section{Built Environment}

Land Development Patterns

- Jand Use Mix

- Density

Urban Design

- Arrangement

- Aesthetic

Transportation System

- Infrastructure

- Performance
* Reverse Coded

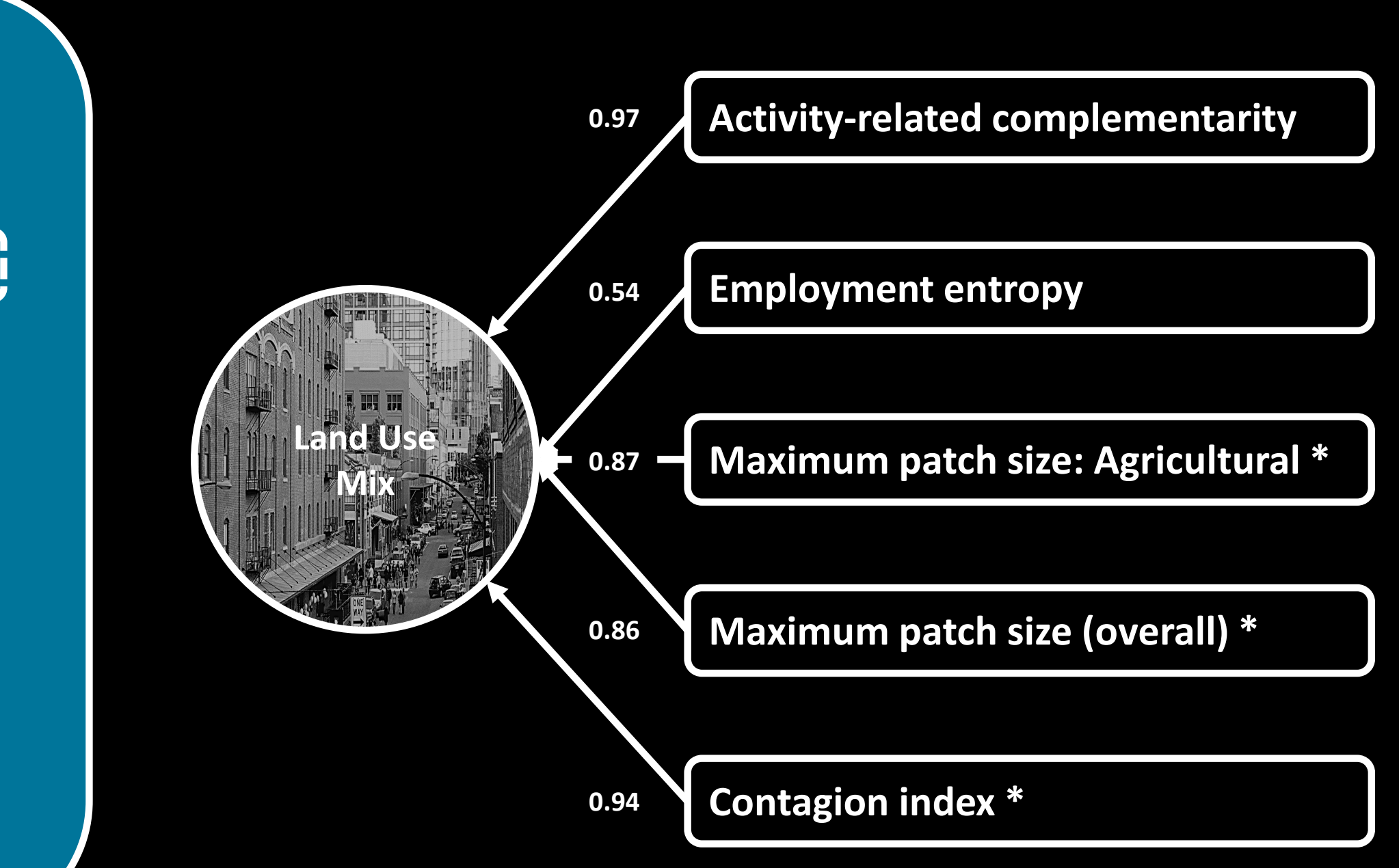

CFA Fit Statistics: CFI: 0.96 | TLl: 0.91 


\section{Confirmatory factor analysis}

\section{Built Environment}

Land Development Patterns

-f Land Use Mix

1

- Density

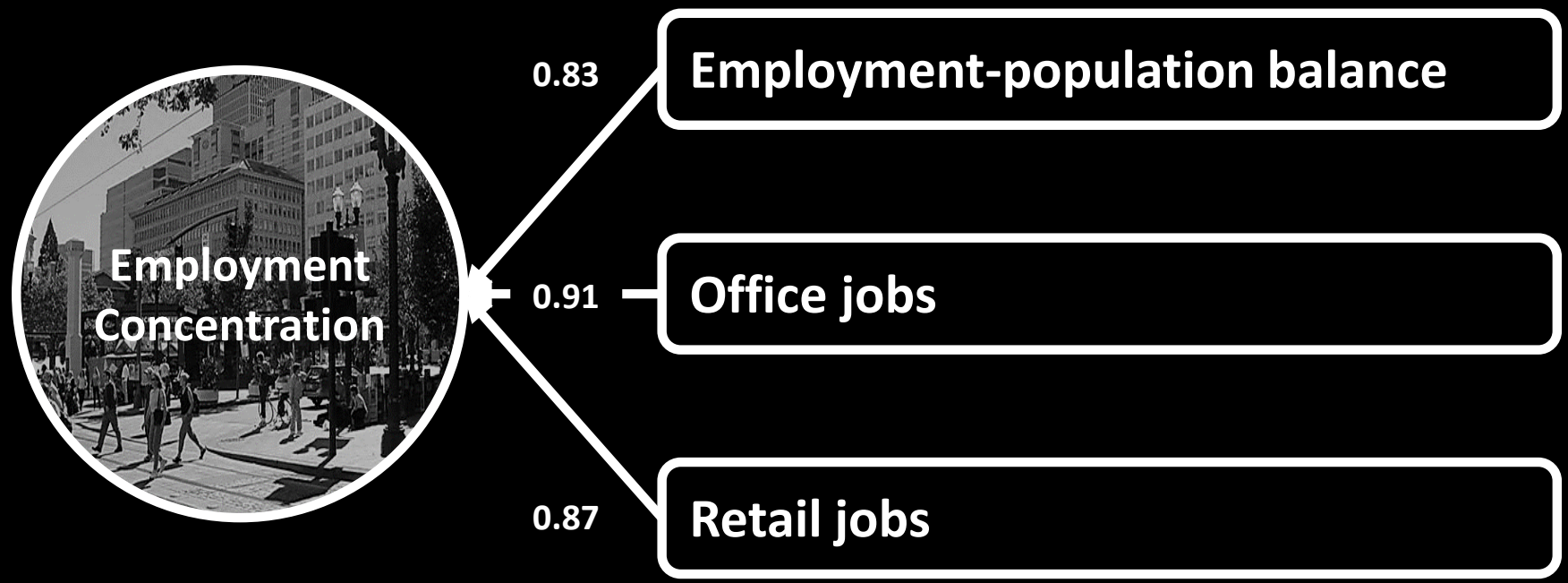

Transportation System

- Infrastructure

- Performance

CFA Fit Statistics: CFI: 0.99 | TLl: 0.99 


\section{Confirmatory factor analysis}

\section{Built Environment}

Land Development Patterns

- \{ Land Use Mix

- Density

Urban Design

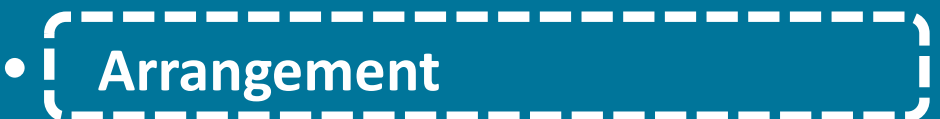

- Aesthetic

Transportation System

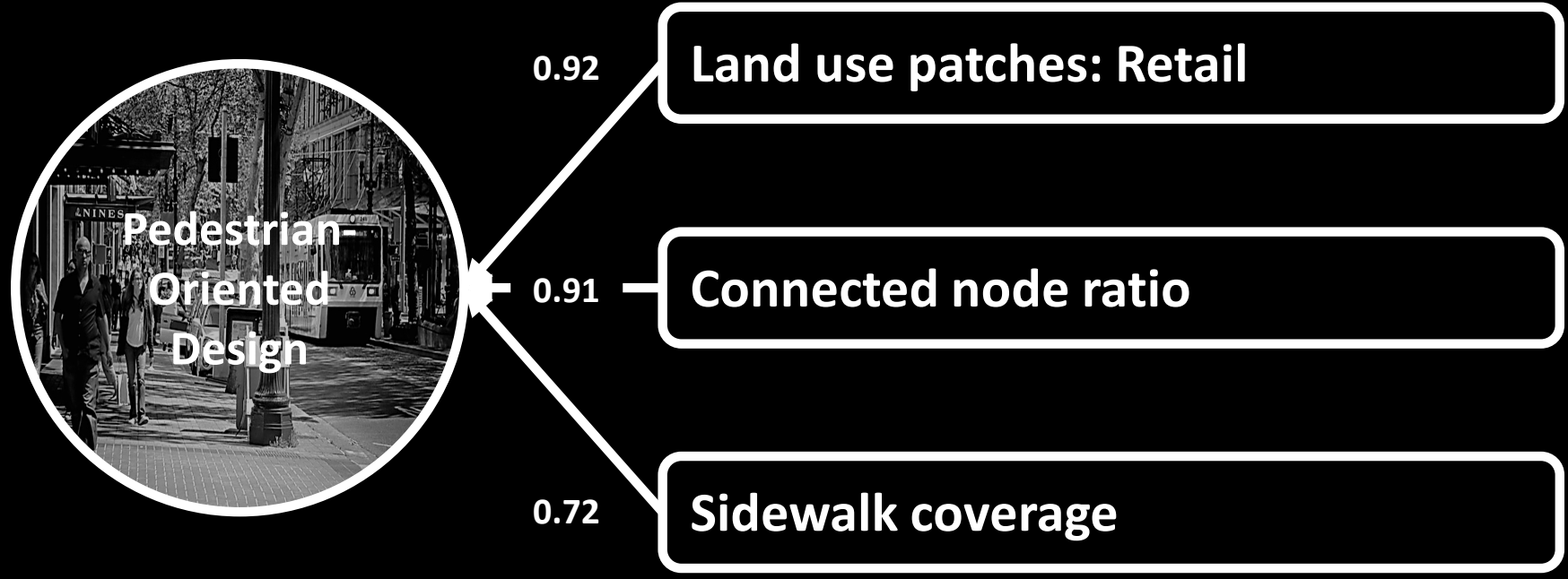

- Infrastructure

Performance

CFA Fit Statistics: CFI: 0.99 | TLI: 0.99 
Structural equation model
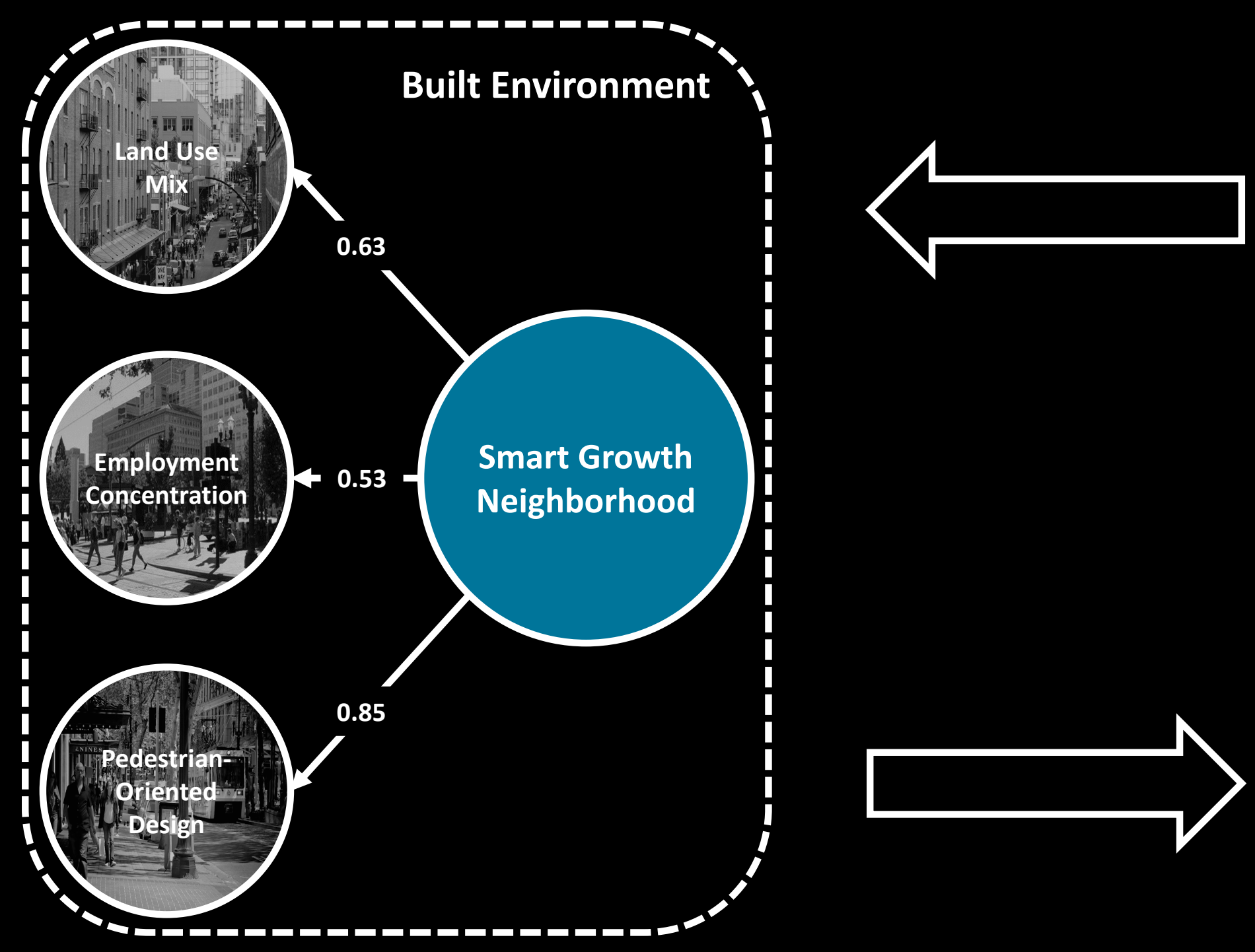

\section{Sociodemographic and Economic}

Number of children under 6 years

Number of children 6 years or older

Number of adults

Non-related household

Annual household income

Number of household workers

Oldest household member

Highest household educational attainment

Vehicles per licensed adult

Transit passes per adult

Bikes per person 6 years or older
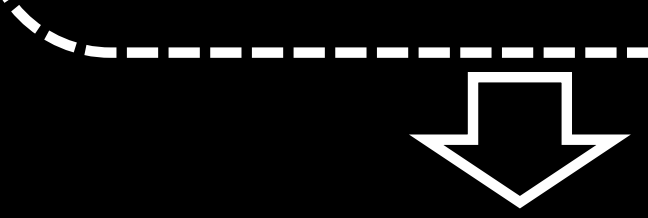

Travel Behaviors and Patterns

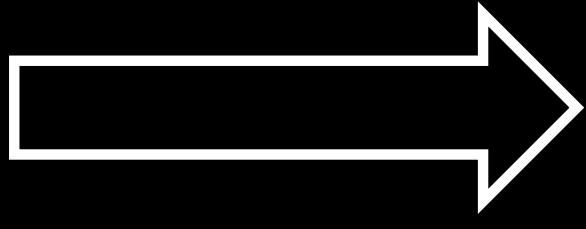

SEM Fit Statistics: CFI: 0.85

TLI: $\mathbf{0 . 8 0}$ | RMSEA: 0.08

Trip Distance

Walk Mode:

Transportation

Walk Mode:

Discretionary 


\section{Results: Walk for transportation purposes}

Outcome: Household-level decision to participate in $\geq 1$ home-based walk trip for transportation purposes

\begin{tabular}{lccc}
\hline Indicator Name & Direct Effect & p-value & Total Effect \\
\hline Number of children under 6 years & 0.04 & 0.05 & 0.04 \\
Number of children 6 years or older & 0.15 & 0.00 & 0.15 \\
Number of adults & 0.10 & 0.00 & 0.07 \\
Annual Income: $\$ 50,000$ to \$99,999 & -0.06 & 0.04 & -0.06 \\
Annual Income: $\$ 100,000$ or more & -0.08 & 0.01 & -0.11 \\
Household workers: 3 or more & -0.05 & 0.01 & -0.05 \\
Education: Graduate degree & 0.05 & 0.10 & 0.09 \\
Vehicles per licensed driver & -0.05 & 0.00 & -0.11 \\
Transit passes per adult & 0.00 & 0.90 & 0.01 \\
Bikes per person 6 years or older & 0.03 & 0.04 & 0.06 \\
\hline Smart Growth Neighborhood & $\mathbf{0 . 2 2}$ & $\mathbf{0 . 0 0}$ & $\mathbf{0 . 2 6}$ \\
\hline
\end{tabular}


Outcome: Household-level decision to participate in $\geq 1$ home-based walk trip for discretionary purposes

\begin{tabular}{lccc}
\hline Indicator Name & Direct Effect & p-value & Total Effect \\
\hline Number of children under 6 years & -0.02 & 0.34 & -0.02 \\
Number of children 6 years or older & 0.06 & 0.01 & 0.06 \\
Number of adults & 0.08 & 0.00 & 0.05 \\
Annual Income: $\$ 50,000$ to $\$ 99,999$ & 0.03 & 0.24 & 0.01 \\
Annual Income: $\$ 100,000$ or more & 0.01 & 0.84 & -0.01 \\
Household workers: 3 or more & -0.04 & 0.03 & -0.04 \\
Education: Graduate degree & 0.05 & 0.09 & 0.07 \\
Vehicles per licensed driver & -0.02 & 0.12 & -0.07 \\
Transit passes per adult & -0.03 & 0.04 & -0.02 \\
Bikes per person 6 years or older & 0.02 & 0.27 & 0.04 \\
\hline Smart Growth Neighborhood & $\mathbf{0 . 1 5}$ & $\mathbf{0 . 0 0}$ & $\mathbf{0 . 1 7}$ \\
\hline
\end{tabular}




\section{Study contributions and potential implications}

- Introduced second-order construct of smart growth reflecting three key tenets

- Provided planners an identified set of indicators reflecting built environment efficiencies

- Guide land development discussion away from contentious debates focused on density

- Demonstrated link between smart growth residential environments and walking

- Strong direct and total effect on household-level choice to participate in a walk trip

- Highlight continued prospect of smart growth policies facilitating more physical activity

\section{Next steps}

- Additional non-built environment variables and complexity to SEM analysis

- Sociodemographic and economic characteristics as formative construct

- Hierarchical framework to model individual-level travel behaviors

- Further attention to choice of geographic scale used to operationalize indicators 


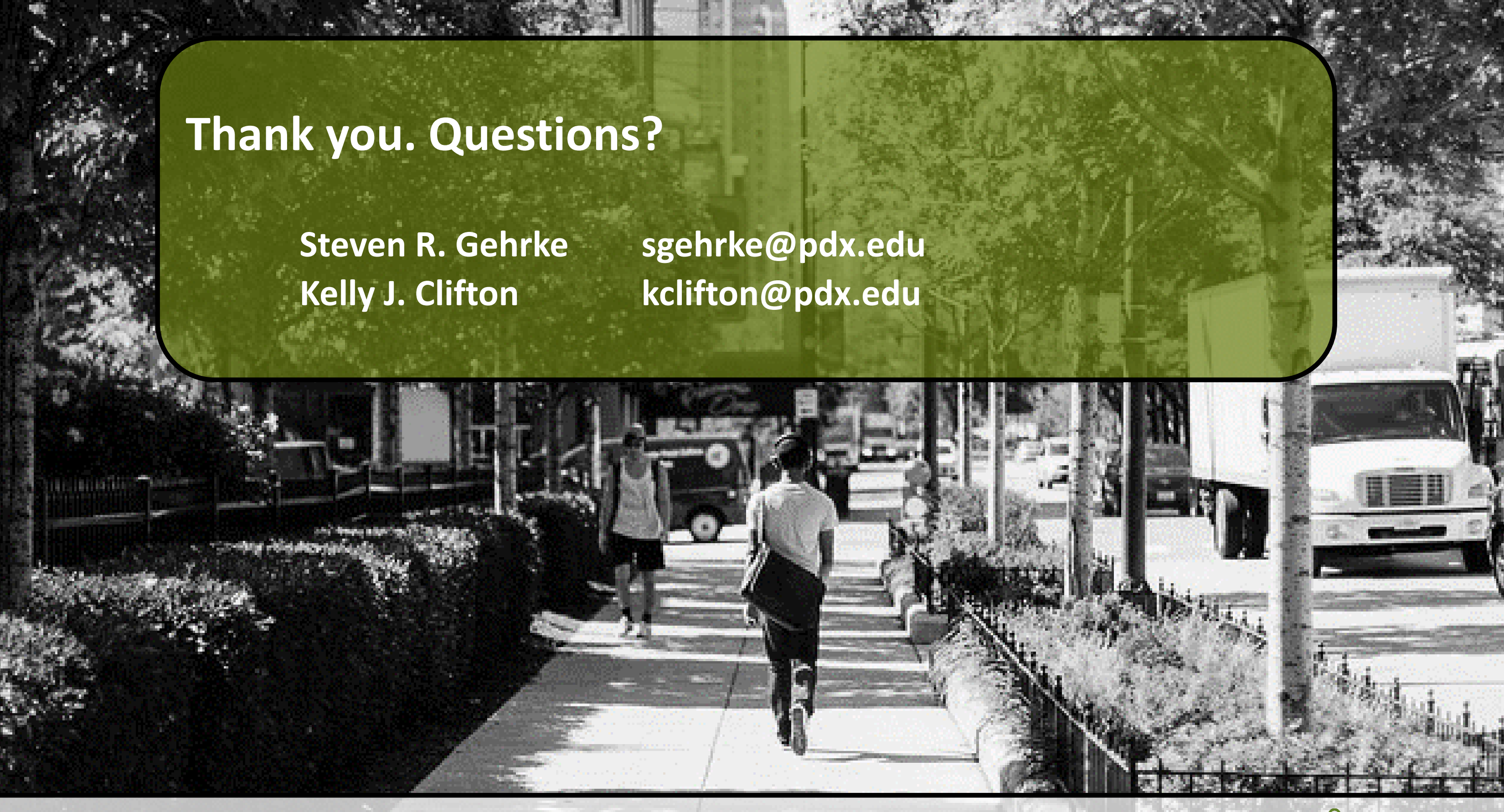

